\section{Myasthenia gravis due to hydroxychloroquine}

\author{
O. Varan, H. Kucuk, A. Tufan \\ Division of Rheumatology, Department of Internal Medicine, Gazi University Faculty \\ of Medicine, Ankara, Turkey
}

Tisese o the Editor:

Myasthenia gravis is a neuromuscular disease accompanied by muscle weakness due to the development of autoantibodies against the acetylcholine receptor (AChr). Hydroxychloroquine is an antimalarial drug used to treat connective tissue diseases. Clinicians should be careful when treating patients with neuromuscular diseases with hydroxychloroquine (1). This paper presents a patient diagnosed with myasthenia gravis due to hydroxychloroquine.

A 29-year-old woman was being followed with a diagnosis of systemic lupus erythematosus. While in remission, she was admitted to the hospital complaining of weakness. She had been taking hydroxychloroquine.

She stated that she felt sleepy, had difficulty keeping her eyes open, and that her eyelids were closing. She also could not keep her arms raised for long periods and had difficulty carrying things.

She did not complain of double vision, difficulty swallowing, or shortness of breath. A neurological examination revealed no significant facial asymmetry and her lower and upper extremity motor and sensory examinations were normal. Cerebellar tests were normal. Electromyography showed no neuromuscular transmission disorder. Thorax computed tomography was consistent with a thymic remnant in the anterior mediastinum. AChr antibody was administered for suspected myasthenia gravis.

The patient's AChr level was $1.1 \mathrm{nmol} / \mathrm{L}$. On neurology consultation, the patient was diagnosed with myasthenia gravis and hydroxychloroquine treatment was stopped. The level of AChr antibody was 0.8 and $0.77 \mathrm{nmol} / \mathrm{L} 2$ and 4 months after with- drawing hydroxychloroquine, respectively, and the patient's complaints had decreased. Therefore, the patient was considered to have myasthenia gravis due to hydroxychloroquine.

Cases of myasthenia gravis due to chloroquine and hydroxychloroquine have been reported. Schumm et al. (2) reported a case of myasthenia gravis due to chloroquine intake, with symptomatic recovery and the disappearance of AChr antibody after withdrawing chloroquine.

In a series of 17 patients with coexisting systemic lupus erythematosus and myasthenia gravis, Jallouli et al. (3) found that the latter disease was due to hydroxychloroquine in 8 patients; no recovery was observed in 3 of 4 patients in whom hydroxychloroquine was withdrawn.

While our patient recovered symptomatically after withdrawing hydroxychloroquine, she was still positive for antibody. In conclusion, a diagnosis of myasthenia gravis should be considered when neuromuscular symptoms develop in patients using hydroxychloroquine.

The authors declare no conflict of interest.

\section{REFERENCES}

1. Luo J, Lindstrom J. AChR-specific immunosuppressive therapy of myasthenia gravis. Biochem Pharmacol. 2015; 97: 609-19.

2. Schumm F, Wietholter H, Fateh-Moghadam A. Myasthenia syndrome during chloroquine treatment (author's transl). Dtsch Med Wochenschr. 1981; 106: 1745-7. [Article in German]

3. Jallouli M, Saadoun D, Eymard B, Leroux G, Haroche $\mathrm{J}$, et al. The association of systemic lupus erythematosus and myasthenia gravis: a series of 17 cases, with a special focus on hydroxychloroquine use and a review of the literature. J Neurol. 2012; 259: 1290-7.
Corresponding author:

Ozkan Varan

Division of Rheumatology, Department of Internal Medicine

Gazi University Faculty of Medicine

06100 Ankara, Turkey

E-mail:ozkanvaran84@gmail.com 